\title{
PROPERTIES OF NEUTRAL HYDROGEN CLOUDS
}

\author{
ULRICH J.SCHWARZ and HUGO VAN WOERDEN \\ Kapteyn Astronomical Institute, University of Groningen, Groningen, The Netherlands
}

\begin{abstract}
In the region $+19^{\circ}<b<+30^{\circ}, 122^{\circ}<l<143^{\circ}, 21-\mathrm{cm}$ line profiles were analyzed into Gaussian components, and these components combined into clouds, by means of computerized procedures. Maps of some of the clouds so defined are presented, and their properties discussed. Many clouds are elongated and/or irregular in shape. Histograms of cloud parameters are presented and subjected to statistical analysis. There is evidence for tenuous clouds of large angular extent but very low column density $\left(N_{\mathrm{H}}<10^{19} \mathrm{~cm}^{-2}\right)$.
\end{abstract}

\section{Introduction}

This paper is a preliminary report on the results of a determination of interstellar cloud properties in a region at intermediate galactic latitudes. The region $\left(122^{\circ}<l<\right.$ $\left.143^{\circ},+19^{\circ}<b<+30^{\circ}\right)$ was selected because it is relatively free from optical peculiarities, and the structure of $21-\mathrm{cm} \mathrm{H}$ I line profiles found in earlier investigations of the region (van Woerden et al., 1962; Takakubo, 1968) appeared well-defined.

The analysis of hydrogen-line profiles in this region represents an attempt to define interstellar clouds, and determine their properties, by quantitative, computerized methods, consistent over the whole region. The need for information of this kind has been emphasized at a recent IAU Symposium (van Woerden, 1970).

\section{Method of Analysis}

The observations (beamwidth 0.6 , bandwidth $2 \mathrm{~km} \mathrm{~s}^{-1}$, rms noise in $T_{b} 0.18 \mathrm{~K}$ ) cover the $20^{\circ} \times 10^{\circ}$ region with a $1^{\circ} \times 1^{\circ}$ grid. The $21-\mathrm{cm}$ emission line profiles were analyzed into Gaussian components; in determining the 'best' among several possible solutions of one profile (always independently for each position), an improved form of the criterion defined by Kaper et al. (1966, Equation (5.6)) was applied.

Next the profile components were compared with those at surrounding positions $\left(|\Delta l|,|\Delta b| \leqslant 2^{\circ}\right)$; components indistinguishable by Gaussian analysis if placed in the same profile were considered as related, and combined into one 'cloud'; separable components were considered unrelated. The comparisons assess both $\Delta V$ and $\Delta \sigma$ in terms of the velocity dispersion, $\sigma$; the component strength only influences the accuracy of the comparisons. A 'cloud' thus defined is essentially a concentration in $(l, b, V, \sigma)$-space.

The process described furnished a total of 88 clouds in the region. Of these, 10 are believed to belong to large-scale structures, including the intercloud medium; we shall not discuss these in detail in the present context. 

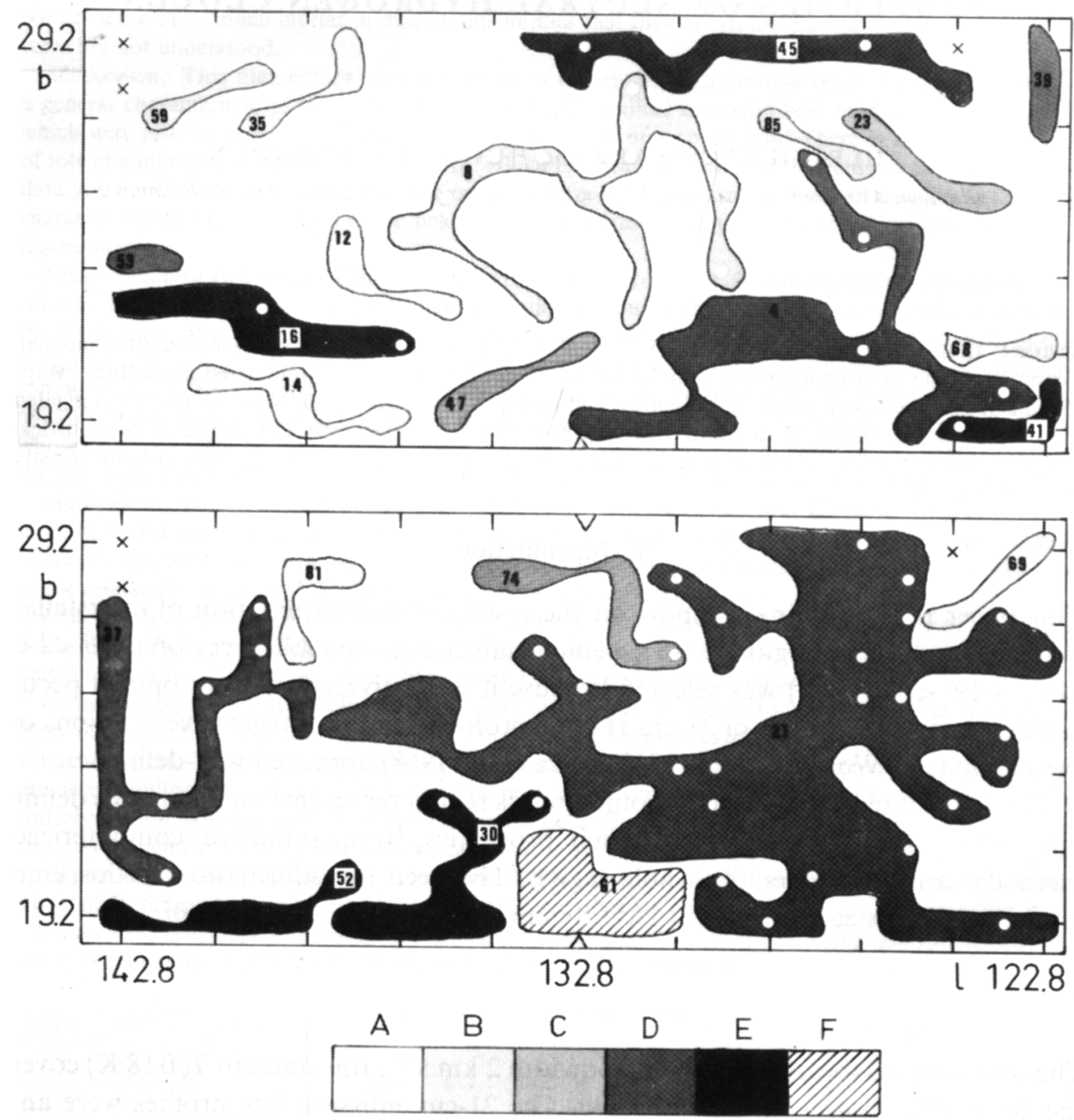

Fig. 1. Positions and shapes of 23 hydrogen clouds. Each cloud is labeled with a number. The grey shades indicate column densities $\bar{N}_{\mathrm{H}}$, as follows:

$$
\begin{aligned}
& \text { Shade } \\
& { }_{0.1}{ }_{0.2}^{\text {B }}{ }_{0.4}{ }_{1}{ }_{\infty}
\end{aligned}
$$

Shade $\mathrm{F}$ indicates clouds with velocity dispersions, $\bar{\sigma}_{0}$, exceeding $10 \mathrm{~km} \mathrm{~s}^{-1}$.

The outline of a cloud encloses all grid positions (and only those) where a profile component is found belonging to the cloud. Dots indicate positions where the component's relationship to the cloud is uncertain Examples: cloud 61 is certainly present at 7 positions $\left(b=+19{ }^{\circ} 2, l=130^{\circ} 8\right.$ and $131^{\circ}: 8 ; b=+20^{\circ} 2, l=130^{\circ} 8$, $131^{\circ} .8$ and $132^{\circ} .8 ; b=+21.2, l=132.8$ and $\left.133^{\circ} 8\right)$ and uncertain at 3 ; it has $\bar{V}_{0}=-17.8 \mathrm{~km} \mathrm{~s}^{-1}$ and $\bar{\sigma}_{0}=29 \mathrm{~km} \mathrm{~s}^{-1}$. Cloud $68\left(\bar{V}_{0}=-39.3 \mathrm{~km} \mathrm{~s}^{-1}, \bar{\sigma}_{0}=1.6 \mathrm{~km} \mathrm{~s}^{-1}, \bar{N}_{\mathrm{H}}=0.035 \times 10^{20}\right.$ atom cm $\left.\mathrm{cm}^{-2}\right)$ has components at $(122.8,+21: 2)$ and $(124.8,+21.2)$, but not at $l=123.8$; this is a 'tenuous cloud' (Section 4$)$.

Note the irregular and filamentary shapes of many clouds.

Crosses indicate 3 unobserved grid positions. 


\section{Cloud Shapes, Parameters and Properties}

(a) SHAPES

Figure 1 shows the shapes for 23 clouds, selected at random from the total. The outlines sketched are determined by the positions where components belonging to a cloud are seen, but they remain arbitrary to some extent, since the beamwidth $(0.6)$ is significantly smaller than the grid spacing (1.0). The most striking characteristics of Figure 1 are the irregular or filamentary shape of many clouds, and the loose, incoherent structure of some. The sample of 23 is not atypical in this respect. Note that some of these structures are not unlike those of parts of supernova remnants or reflection nebulae.

(b) OBSERVED PARAMETERS

For each of the 88 clouds, a number of observed parameters were calculated. For most of these parameters, the statistics are displayed in the histograms of Figure 2. We make the following notes.

(i) The distributions of optical depth $\bar{\tau}_{0}$, column density $\bar{N}_{\mathbf{H}}$ and total hydrogen content $S$ (related to mass, see below) increase steeply towards the lowest values. This is in agreement with earlier findings (e.g., Clark, 1965; Radhakrishnan and Goss, 1972; Heiles, 1967; Field and Hutchins, 1968), but extends them to much smaller values of the parameters.

(ii) The distribution of radial velocity $\bar{V}_{0}$ is surprisingly wide, much wider than is compatible with external velocity dispersions usually assumed (cf. van Woerden, 1967, Table 4). The cause of this discrepancy is under further investigation.

(iii) The distribution of velocity dispersion $\bar{\sigma}_{0}$ peaks between 1 and $3 \mathrm{~km} \mathrm{~s}^{-1}$. This result is closely similar to those obtained in absorption by Muller (1959), Clark (1965) and (also in emission) Radhakrishnan et al. (1972; they give $\Delta V_{1 / 2}=2.35 \sigma$ in Tables 2 and 4). In our earlier study of emission profiles in Orion (van Woerden, 1967, Figure 10 and Table 5), where we followed much the same methods as in the present work, somewhat larger values of $\bar{\sigma}_{0}$ were derived.

(iv) For most clouds, the minor angular diameter $\beta$ (for definition see the caption of Figure 2) is much smaller than the major diameter $\alpha$, in agreement with the filamentary structure noted above. Some clouds may be unresolved across their major diameter.

\section{(c) INTRINSIC PROPERTIES}

The observed parameters $\alpha, \beta, S$ and $\bar{N}_{\mathrm{H}} / \beta$ are related to intrinsic properties via relations involving various powers of the unknown distance, $r$ :

major linear diameter, $a \sim \alpha r$

minor linear diameter, $b \sim \beta r$

hydrogen mass, $M_{\mathrm{H}} \sim S r^{2}$

hydrogen volume density, $n_{\mathrm{H}} \sim \bar{N}_{\mathrm{H}} \beta^{-1} r^{-1}$

Assuming a specific distribution of distances, one may derive from the distributions 

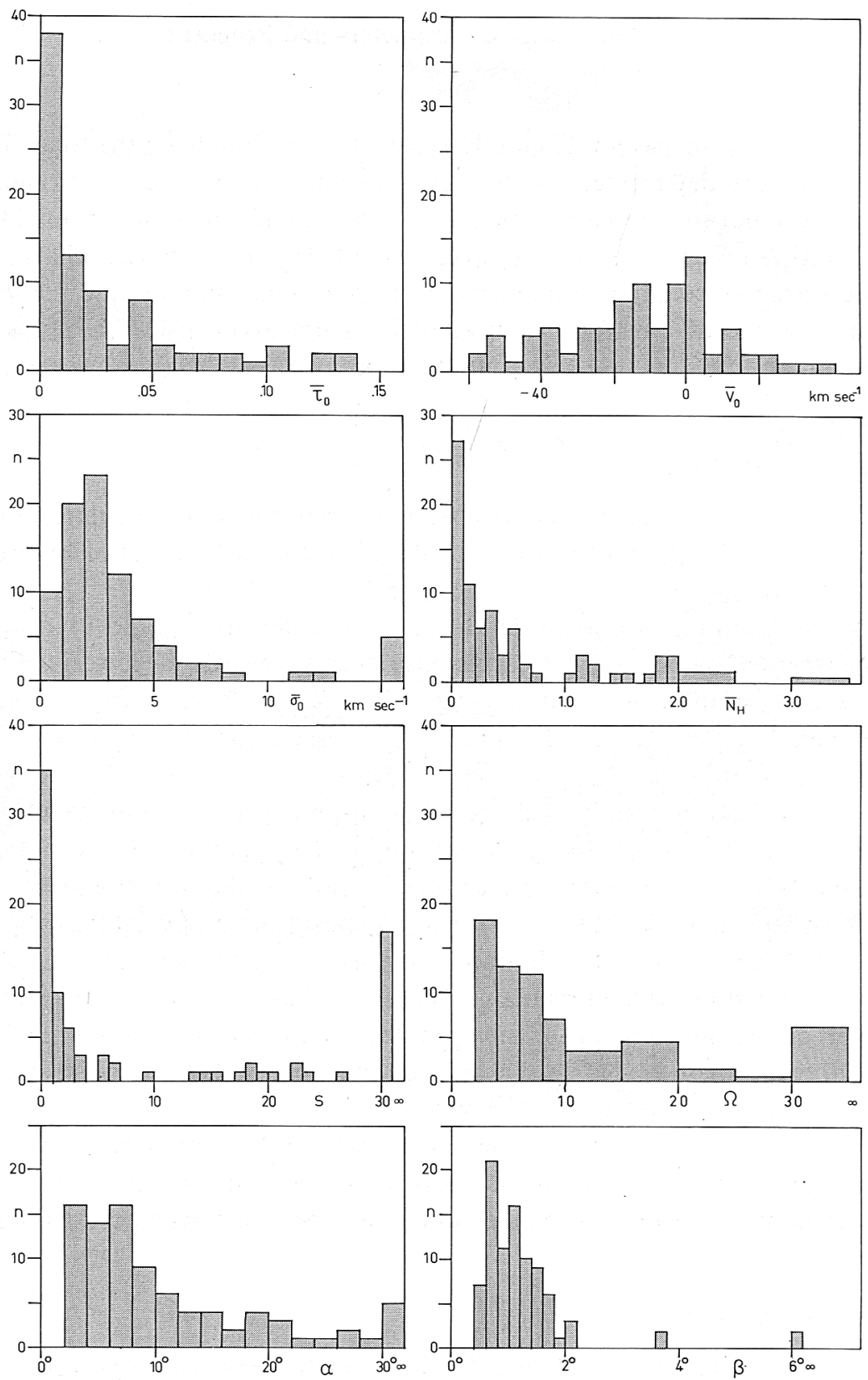

Fig. 2. Histograms of observed cloud parameters:

$\bar{\tau}_{0}$, average peak optical depth (assuming a spin temperature of $125 \mathrm{~K}$ ); $\bar{V}_{0}$, average radial velocity (with respect to LSR); $\bar{\sigma}_{0}$, average velocity dispersion (corrected for instrumental broadening); $\bar{N}_{\mathrm{H}}$, average column density (unit: $10^{20}$ atom cm ${ }^{-2}$ ); $S \equiv \Sigma N_{\mathrm{H}} \equiv \bar{N}_{\mathrm{H}} \times \Omega$ (unit: $10^{20}$ atom cm $\mathrm{cm}^{-2}$ degree $\left.^{2}\right) ; \Omega$, area covered $\equiv$ number of grid positions where cloud is present (unit: degree $^{2}$ ); $\alpha$, major angular diameter; $\beta \equiv \Omega / \alpha$, minor angular diameter.

For clouds reaching the edge of the observed region, the quantities $S, \alpha$ and $\Omega$ are multiplied by 2 . 
of observed parameters those of intrinsic properties. Preliminary results suggest the following power laws:

$$
\psi(a)=C a^{-1 \text { to }-2}, \quad \psi(M)=C M^{-1}, \quad \psi(n)=C n^{-1.5} .
$$

\section{Tenuous Clouds}

A large number of clouds have very small column densities, $\bar{N}_{\mathrm{H}}<0.1 \times 10^{20}$ atom cm $^{-2}$. This finding is due to the high sensitivity of observations and analysis. For the 7 'tenuous clouds' with $\bar{N}_{\mathrm{H}}<0.05 \times 10^{20}$ and $\Omega \geqslant 3$, Table I compares the average parameters with those of Heiles' (1967) cloudlets. The differences are obvious.

TABLE I

Tenuous clouds and cloudlets

\begin{tabular}{|c|c|c|}
\hline & $\begin{array}{l}\text { Average } \\
\text { tenuous cloud } \\
\text { (this paper) }\end{array}$ & $\begin{array}{l}\text { Average } \\
\text { cloudet } \\
\text { (Heiles, 1967) }\end{array}$ \\
\hline Peak brightness temperature, $T_{\mathrm{b} 0}(\mathrm{~K})$ & 0.6 & 7 \\
\hline Column density, $\bar{N}_{\mathrm{H}}\left(10^{20}\right.$ atom $\left.\mathrm{cm}^{-2}\right)$ & 0.044 & 0.3 \\
\hline Hydrogen content, $S\left(10^{20}\right.$ atom $\mathrm{cm}^{-2}$ degree $\left.^{2}\right)$ & 0.28 & 0.6 \\
\hline Velocity dispersion, $\bar{\sigma}_{0}(\mathrm{~km} / \mathrm{s})$ & 1.5 & 0.7 \\
\hline Major diameter, $\alpha$ (degrees) & 7 & \\
\hline Minor diameter, $\beta$ (degrees) & 1 & 0.5 \\
\hline Upper limit to kinetic temperature: $T_{\mathrm{k}}(\mathrm{K})$ & $\leqslant 280$ & $\leqslant 70$ \\
\hline
\end{tabular}

The considerable angular size of these objects results in a very low density - unless they are extremely nearby. A distance of $1 \mathrm{kpc}$ (the expected distance of most of the hydrogen in this direction) would make the sizes $100 \times 20 \mathrm{pc}$, mass $100 M_{\odot}$, density $0.1 \mathrm{~cm}^{-3}$; at $10 \mathrm{pc}$ distance, the sizes are $1 \times 0.2 \mathrm{pc}$, mass $0.01 M_{\odot}$, density $10 \mathrm{~cm}^{-3}$. Equilibrium with an intercloud medium of kinetic temperature, $T_{k} \approx 10000 \mathrm{~K}, n_{\mathrm{H}} \approx$ $\approx 0.17 \mathrm{~cm}^{-3}$ would require for the 'tenuous clouds' $n_{\mathrm{H}} \approx 6 \mathrm{~cm}^{-3}$, thus a distance close to 10 pc. Possible alternatives include the following:

(i) The clouds are at about $1 \mathrm{kpc}$, but their minor diameters are overestimated (unresolved) and densities underestimated; the clouds are then extremely elongated.

(ii) The clouds are in a transient phase, not in equilibrium with their surroundings.

(iii) The kinetic pressure of the intercloud medium is offset by magnetic rather than kinetic pressure inside the clouds.

(iv) The intercloud medium is much cooler than $10000 \mathrm{~K}$.

This work will be submitted in more detail to Astronomy and Astrophysics or its Supplements. 


\section{Acknowledgements}

The observations were done at Dwingeloo, with financial support from the Netherlands Organization for the Advancement of Pure Research (ZWO).

\section{References}

Clark, B. G.: 1965, Astrophys. J. 142, 1398.

Field, G. B. and Hutchins, J.: 1968, Astrophys. J. 153, 737.

Heiles, C. : 1967, Astrophys. J. Suppl. 15, 97.

Kaper, H. G., Smits, D. W., Schwarz, U. J., Takakubo, K., and Woerden, H. van: 1966, Bull. Astron. Inst. Neth. 18, 465.

Muller, C. A.: 1959, in R. N. Bracewell (ed.), 'Paris Symposium on Radio Astronomy', IAU Symp. 9, 360. Radhakrishnan, V. and Goss, W. M.: 1972, Astrophys. J. Suppl. 24, 161.

Radhakrishnan, V., Murray, J. D., Lockhart, P., and Whittle, R. P. J.: 1972, Astrophys. J. Suppl. 24, 15. Takakubo, K.: 1968, Bull. Astron. Inst. Neth. 20, 107.

Woerden, H. van: 1967, in H. van Woerden (ed.), 'Radio Astronomy and the Galactic System', IAU Symp. 31, 3.

Woerden, H. van: 1970, in H. J. Habing (ed.), 'Interstellar Gas Dynamics', IAU Symp. 39, 369.

Woerden, H. van, Takakubo, K., and Braes, L. L. E.: 1962, Bull. Astron. Inst. Neth. 16, 321.

Ulrich J. Schwarz

Hugo van Woerden

Kapteyn Astronomical Institute,

University of Groningen,

Postbus 800,

Groningen 8002, The Netherlands 